\title{
VACUUMFORMED THERMOPLASTIC Aligners IN ORTHODONTICS
}

\author{
II \\ SZUHANEK C. \& GRIGORE A. \\ II
}

Abstract: Thermoplastic aligners are frequently used in orthodontics when it comes to the correction of mild malocclusions These appliances are very popular because they provide an esthetic and comfortable alternative to conventional treatment due to their translucency and reduced thickness. The thermoplastic aligners are well fitted and offer good treatment results if they are worn properly. Aligners have less social impact than braces. The foils that are used for the vacuumforming process are made out from clear thermoplastic material, such as polyethylene or PC. The polyethylene has enough stiffness to provide the desired tooth movements and helps patients to maintain a good oral hygiene. Aligners must be changed every two or three weeks until the final result is reached. Material thickness can also be increased during the treatment period in order to assure stiffness and reduce the total treatment time.

Key words: vacuumformed aligners, thermoplastic, polyethylene, biomechanics, orthodontics
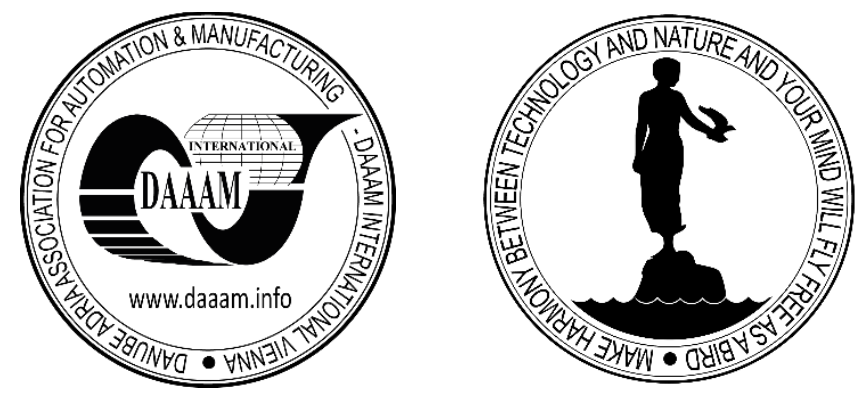

Authors' data: S.L. Dr. Szuhanek C[amelia], Dr. Grigore A[delina], Faculty Of Dental Medicine, University Of Medicine And Pharmacy "Victor Babeș" Timișoara, România, Piața Eftimie Murgu nr. 2, Timișoara, România, cameliaszuhanek@umft.ro, grigoreadelina@yahoo.

This Publication has to be referred as: Szuhanek, C[amelia] \& Grigore, A[delina] (2016). Vacuumformed Thermoplastic Aligners in Orthodontics, Chapter 27 in DAAAM International Scientific Book 2016, pp.307-314, B. Katalinic (Ed.), Published by DAAAM International, ISBN 978-3-902734-09-9, ISSN 1726-9687, Vienna, Austria

DOI: $10.2507 /$ daaam.scibook.2016.27 
Szuhanek, C. \& Grigore, A.: Vacuumformed Thermoplastic Aligners in Orthodontics

\section{Introduction}

Nowadays, adult patients seek aesthetic alternatives to conventional orthodontic treatment. Progressive dental alignment can be done with a series of translucent thermoplastic aligners.

The basis of aligners' technology was first introduced in 1971 when Pontiz discovered the first thermoplastic materials. Minor dental corrections were first achieved with the use of Essix retainers. These retainers were introduced by Dr. John Sheridan. His technique was very simple: on an in-office set-up, several misaligned teeth were repositioned with the use of dental wax and a vacuumformed aligner was then manufactured over the plaster model which represented the dental set-up. The idea of progressive aligners for minor corrections was introduced by McNamara. Stripping or IPR (interproximal reduction of enamel) was performed if needed (orthodontic patients with dental crowding).

The first invisible orthodontic system was developed in 1999 (Invisalign; Align Technology). The system combines the CAD/CAM technology with a series of more complex steps. The aligners can be made out of PETg, EVA or PC materials. One of the most important features of this system is represented by the digital set-up of the case and the digital planning of the treatment (quantity of dental movements, type of dental movement, the number of the required aligners for each case, the needed quantity of interproximal enamel reduction, number of activations, etc.). All patients can visualize, with the use of a 3D software programme (ClinCheck), the predicted treatment outcome. This advantage can increase the patient's compliance for more difficult cases. The orthodontist can also visualize the predicted tooth movement at each stage of the treatment. The newest ClinCheck programmes also consider the biological limitations of each case and can also include the position of the roots.

Align Technology consists of a series of vacuumformed invisible appliances. Each aligner can assure a minor dental movement $(0,25 \mathrm{~mm})$. The needed number of aligners depends on the severity of the case.[Avesh Sachan, 2013]

Several other systems were developed since then: Clear Aligner, Clear Correct, Nuvola, Fantasmino, My Clear Brace, etc. These new systems also include new types of materials (PVC) that can reduce the optimal daily wear time of the appliances from 20 hours to 14 hours/day due to their plastic deformation characteristics when exposed to moderate orthodontic forces [Ercoli F., 2014].

Material thickness may also vary among the systems. Clear Aligner uses several foils: $0,5 \mathrm{~mm}$ thickness for the first week (first treatment step), 0,625 $\mathrm{mm}$ for the second week (2nd treatment step), 0,75 $\mathrm{mm}$ for the $3 \mathrm{rd}$ week (3rd treatment step). Every three weeks a new series of aligners is manufactured (consisting of three steps). Ortho Analyzer is the 3D software that Clear Aligner uses. All dental movements or attachments can be visualized by the orthodontist before proceeding to the manufacturing of the aligners. Complex cases require a considerable number of steps.[Echarri P., 2013] The predicted dental movements are displayed individually for each tooth: rotations, angulation, inclination, stripping quantity, etc. (table 1). We selected a clinical case to emphasize the importance of the digital panning phase (table 1, figure 1). 


\begin{tabular}{|c|c|c|c|c|}
\hline Tooth number & Rotations & Angulation & Inclination & $\begin{array}{c}\text { Stripping } \\
\text { quantity }\end{array}$ \\
\hline 2.1 & $-4,6$ & $-0,1$ & 1,4 & 0 \\
\hline 2.2 & $-17,5$ & 0,5 & 1,3 & 0 \\
\hline 2.3 & 35,4 & 4,7 & 6,4 & 0 \\
\hline
\end{tabular}

Tab. 1. Predicted orthodontic tooth movement for 2.1, 2.2, 2.3

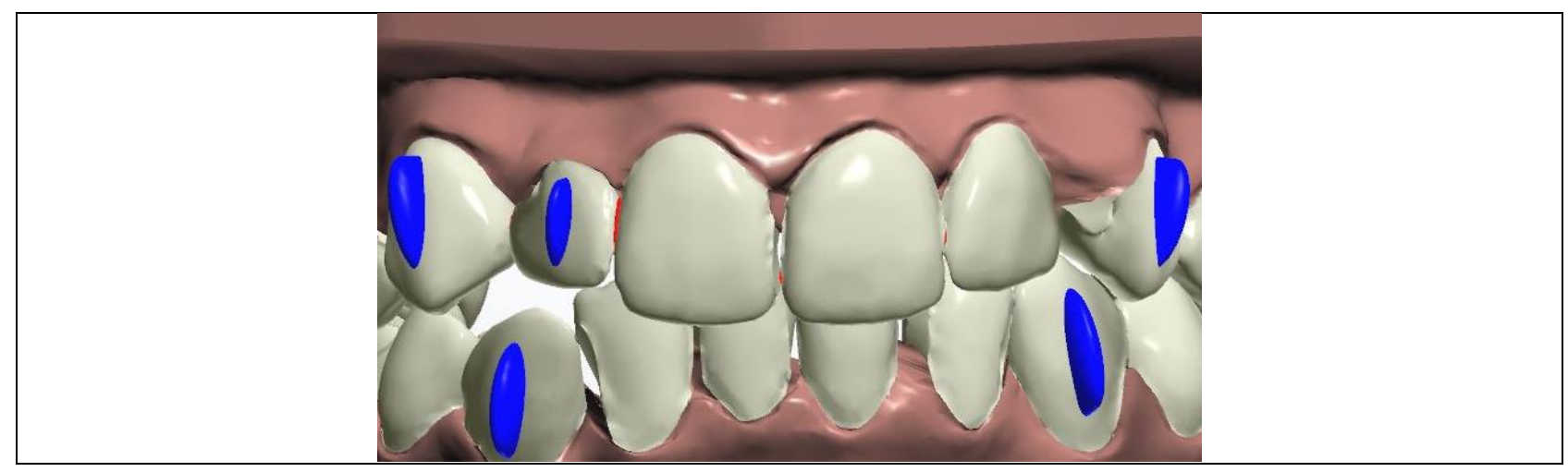

Fig. 1. The digital set-up with the included attachments

Thickness reduction should be considered during the manufacturing process of the vacuum formed aligners. There are only a few studies that take into account this feature. Although the foils are sensitive to heat, no major thickness reduction was noticed after the fabrication of the aligners [Szuhanek C., 2015].

\section{Indications and limitations of the invisible orthodontic systems}

Although the invisible orthodontic systems are very popular among adults, they have specific indications and certain limitations. Dental intrusions, deep-bite cases, minor tooth rotations, small antero-posterior discrepancies and crowding cases $(<4$ $\mathrm{mm}$ ) can be easily resolved with the use of the vacuumformed appliances. Even more difficult dental movements (torque, molar uprighting) can be almost entirely corrected if resin attachments are added on the tooth surface or included in the aligner's design. Another advantage of the aligner therapy consists in the possibility of improving a sleep apnea problem. By aligning the teeth and by expanding the dental arches, the obstruction of the air passage way is no longer an issue. Class elastics and aligner therapy could also improve the position of the mandible, clearing the air passage way.

Regarding periodontal health, aligners seem to be more appropriate. Patients are able to remove the appliance when they eat, drink or brush. Several studies indicate that the periodontal status can improve after active orthodontic treatment with aligners due to the correction of crowding. Stripping can also improve the periodontal status by restoring the adequate space for the gingival papilla. Patients that present inesthetic black triangles can also benefit from stripping therapy. Reshaping triangular teeth can reduce the presence of black triangles. Plaque accumulation is also lower with aligner therapy when compared to braces. Several studies show that after 12 months of active treatment, the values of the plaque index and gingival bleeding index where about 5 times higher in patients with conventional straight-wire appliances than aligner cases. [Marzieh K., 2013] 
The limitations, from our point of view, refer to more complex orthodontic objectives that are difficult to achieve with invisible systems alone: open bite cases, distalization more than $4 \mathrm{~mm}$, a stable transverse expansion of the arch more than 3-4 $\mathrm{mm}$ (even if the buccal corridors are improved), crowding or spacing more than $4 \mathrm{~mm}$, a class II/III skeletal pattern and dental extrusions. Extraction cases are also controversial with the use of the orthodontic invisible systems [Melsen B., 2014]. Premolar extraction cases are very difficult to manage. Space closure without tipping is almost impossible. Lower incisor extractions are indicated only in crowding cases $(<5 \mathrm{~mm})$ but translational movements of the teeth are hard to achieve, even with attachments. We recommend oral buttons (added on the aligners) to improve the results and top prevent tipping [Szuhanek C., 2010].

\section{Advantages of the orthodontic invisible systems}

Even if some limitations are to be considered, several advantages must not be neglected. Root resorption is a high debatable subject in the orthodontic literature. The invisible orthodontic systems are said to have less iatrogenic effects such as OIIRR (orthodontically induced inflammatory root resorption). There could be two reasons for that. The first one is the moderate orthodontic forces that are applied to the dental structures with the use of invisible aligners. The second reason refers to the severity of the malocclusion. Is is known that the greater the skeletal discrepancy, the greater the risk of root resorption [Daljit S., 2011]. Class II and class III skeletal patterns are not usually resolved with aligners, so in class I patients the risk of root resorption is quite low. Genetic predisposition or environmental factors should also be considered. Other advantages are related to esthetics, comfort and periodontal health. [Melsen B., 2014]

\section{Disadvantages of the invisible orthodontic systems}

One of the most important requirements of these systems is a good impression. Impressions are taken using polyvinylsiloxane materials. This requires additional chair time. The accuracy of the impressions must be $<3$ microns. Otherwise, the scanning of the dental impressions becomes inaccurate.

Another disadvantage of these systems refers to achieving parallel roots at the end of the orthodontic treatment. Currently, the design of the attachments is still improving in order to achieve better parallelism of the roots. With more complex and sophisticated resin attachments, extraction cases will also be easier to solve in the future.

Skeletal discrepancies more than $2 \mathrm{~mm}$, using class elastics, are also hard to correct. Other methods, such as acrylic inclined planes added to the invisible aligners could work, but only in growing patients with a developing class II or class III malocclusion.

Lack of patient cooperation could also lead to treatment failure. These removable aligners must be worn at least 14-20 hours/day. 


\section{Resin attachments}

The need for resin attachments/other metallic accessories [Szuhanek, 2010] is dictated by some of the complex orthodontic movements or situations, such as incisor and molar torque, extrusions, cylindrical teeth, severely rotated teeth, extraction cases, translational movements. Attachments can vary a lot, depending on the required dental movements. The most common types of attachments are ellipsoid and rectangular. These attachments improve the aligner's retention but they must be added from the beginning of the treatment. The resin attachment can be parallel with the occlusal plane or placed vertically. They can also be placed on the buccal side of the tooth. [Avesh Sachan, 2013]

If the orthodontist decides to include attachments in the treatment planning, the digital set-up of the case (a 3D software) will also show the exact position of these attachments (table 2). A thin template $(0,25 \mathrm{~mm}$ thickness) will be delivered to the dental office in order to reproduce the desired attachment on the tooth surface.

\begin{tabular}{|c|c|}
\hline Required movement & Attachment placement \\
\hline Rotations & Vertical \\
\hline Extrusion & Parallel to the occlusal plane \\
\hline Incisor torque & Parallel to the incisal edge \\
\hline Bodily movements & Vertical \\
\hline Intrusion & Parallel to the occlusal plane \\
\hline Extraction cases/space closure & Vertical attachments on the adjacent \\
& teeth \\
\hline Tipping & Horizontal \\
\hline
\end{tabular}

Tab. 2. Attachment placement.

\section{Material and methods}

We selected an easy way of treating orthodontic patients in our dental office. A more rapid and less expensive alternative to the invisible orthodontic systems is represented by the manufacturing of in-office set-ups, with the use of a good dental technician. A manual set-up can be designed, without wasting time with the delivery of the impressions, and the digital planning of the cases. All the teeth that require a minor correction $(0,2-0,3 \mathrm{~mm})$ must be sectioned from the plaster model and repositioned with the use of a light-cured flowable composite. The new positions of the teeth must be checked in relation to the other dental arch.

After the dental set-up is ready, the vacuum forming machine must be ready. Before the manufacturing of the aligner, an Isofolan foil must be used, in order to have a minor distance between the set-up and the appliance. The Isofolan foil is manufactured over the dental set-up with the use of the Ministar/Biostar machine. This thin layer $(0,01 \mathrm{~mm})$ will provide comfort but will still maintain enough tension in order to achieve the desired dental movement. 
After the Isofolan foil is manufactured, the foil for the aligner is prepared. The thickness may vary between $0,5 \mathrm{~mm}-0,8 \mathrm{~mm}$. The vacuumforming machine must be heated. The heated foil is then applied over the dental set-up and the Isofolan foil (that acts like a separating thin layer between the teeth and the appliance). After the appliance is obtained, it must be polished (figure 2).

Some of the crowding cases require arch expansion, previous to the aligning phase. Therefore, the first aligner must include an expansion screw (Clear Aligner screws). After obtaining the required space, other dental corrections can be implemented. Usually, a midline diastema dictates the beginning of the next step (figure 3).
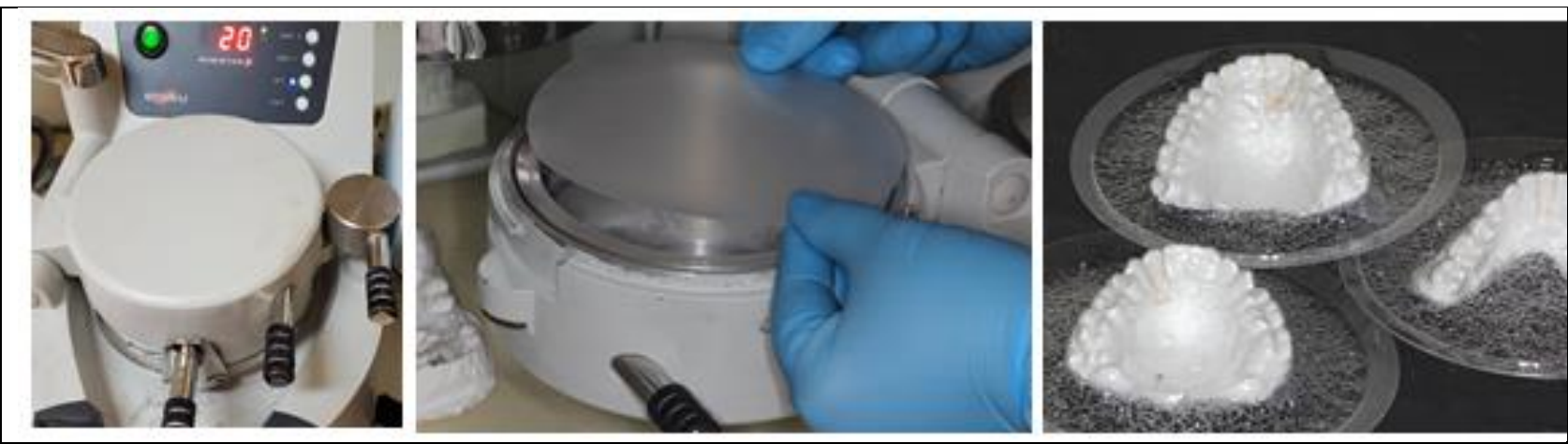

Fig. 2. The manufacturing process of the vacuumformed appliances (the vacuum forming machine, the Isofolan foil, the aligners before the excess removal and polishing phase)

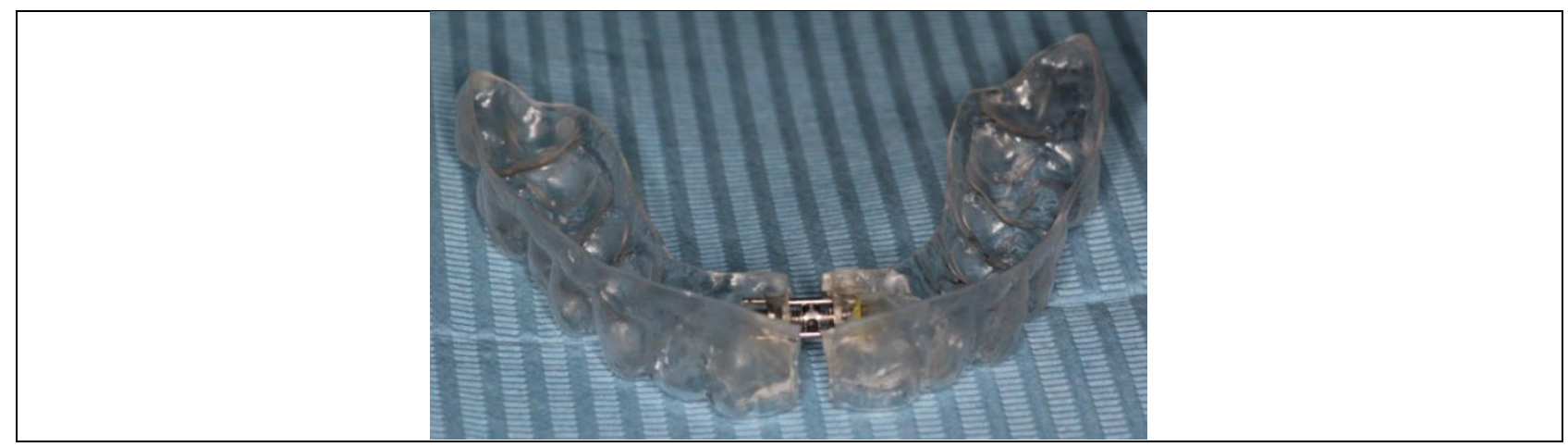

Fig. 3. A vacuum formed aligner with a midline expansion screw

After enough space was obtained, another set-up can be made with the required dental corrections (in our case: derotation of the incisors and the right canine). The aligner with minor corrections $(0,2 \mathrm{~mm})$ was manufactured (figure 4$)$.

Fig. 4. Vacuum formed aligner with the repositioning of 1.1, 2.1, 1.2, 1.3 
Other efficient aligners can be made directly in the dental office. In growing patients, acrylic bite planes can be added to the upper vacuumformed aligner (class II patients) or to the lower one (class III patients) in order to reposition the mandible or to correct a deep bite case. Using the same protocol, a dental technician can easily manufacture different acrylic components (bite planes, bite ramps, etc.).

Another desired objective was to determine if the in-office aligners are efficient. A dental cast was selected. A manual set-up, with the corresponding aligner, was manufactured. The original arch was coloured with red occlusion spray. The aligner was then applied, using a moderate force, on the dental cast(figure 5). The appliance was then removed and the pressure points were checked. The areas where the occlusion spray was no longer well impregnated, were the areas where the aligner was well fitted and where the orthodontic forces were the highest (figure 6).

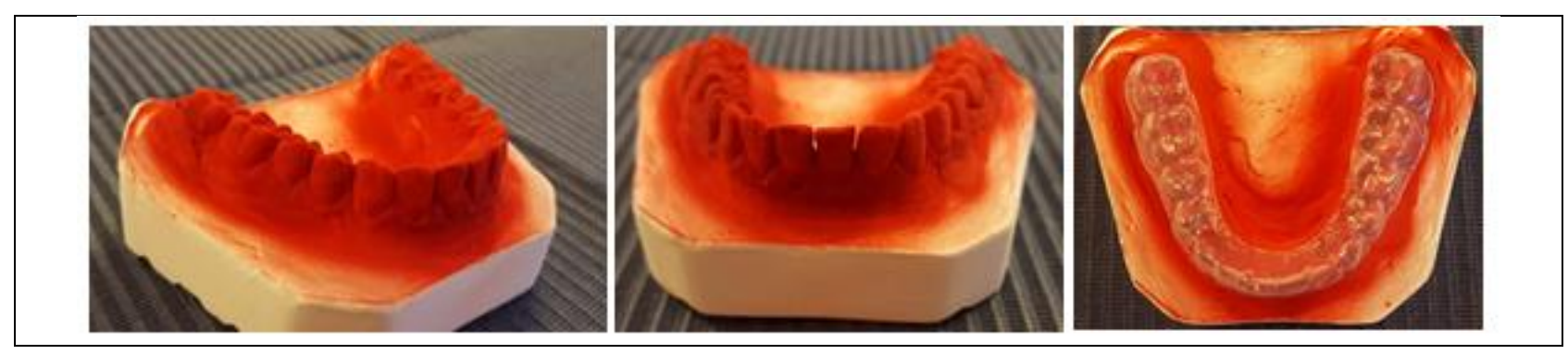

Fig. 5. The original dental arch and the vacuum formed aligner (covering the occlusion spray)

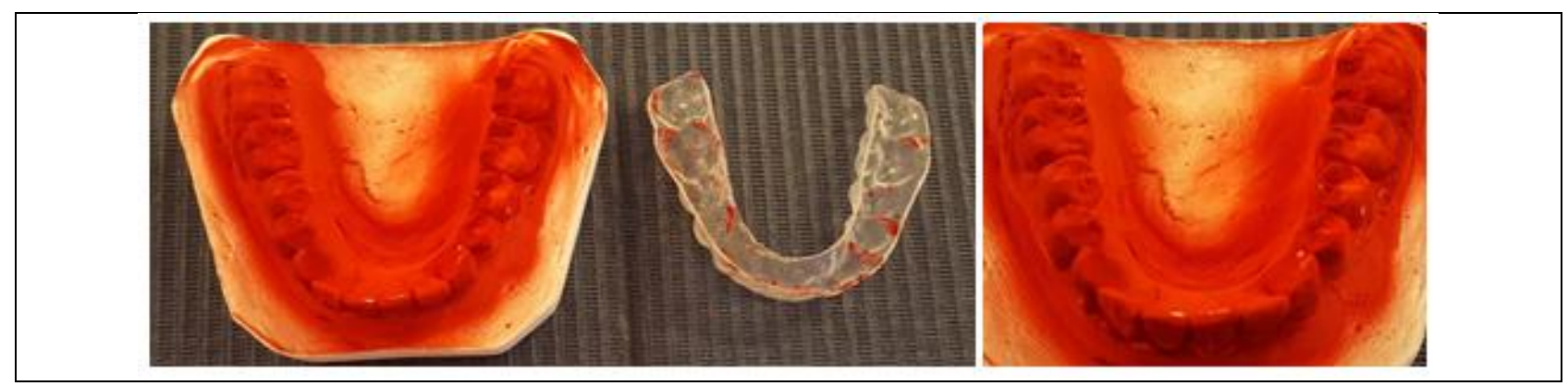

Fig. 6. The pressure areas are evident (the lower incisors and the rotated premolars)

\section{Results and discussions}

At a moderate load, the highest pressure point was found in the lower incisor area (the exact teeth that were included in the manual set-up). This represents the expected results for our case. The most interesting fact is that the rotated premolars were also exposed to orthodontic forces/pressure, even though these teeth were not repositioned. This was a pleasant discovery, since the position of the premolars also requires corrections.Considering the elastic deformation of the appliance, the thickness and the material, there must be other pressure areas besides the ones included in the set-up, such as the pressure areas that we observed in the premolar region, in order to obtain a well fitted aligner and a good distribution of the forces. More information is required, through-out a finite element analysis, to confirm these findings or to compare the results. 
Szuhanek, C. \& Grigore, A.: Vacuumformed Thermoplastic Aligners in Orthodontics

\section{Conclusion}

Adult patients will always lean more towards esthetic alternatives to conventional orthodontic straight-wire appliances. The popularity of the vacuum formed aligners is increasing, demanding constant improvements, even for more complex malocclusions.

The invisible orthodontic systems could be improved if more information is obtained from a clinical point of view. A finite element analysis could offer a more elaborate result regarding the distribution of the orthodontic forces among the periodontal fibers, roots and maxillary bones. This will lead to more esthetic, efficient, comfortable aligners, with reduced thickness, capable of resolving a more complex dental malocclusion.

\section{References}

Avesh Sachan, Invisalign-A modern approach to orthodontic treatment, Lambert Academic Publishing, 2013

Echarri P. Clear Aligner $1^{\text {st }}$ Edition, Ripano S.A., Madrid, 2013

Ercoli F. et al,A comparative study of two different clear aligner systems, Prog Orthod. 2014 May 2;15(1):31. doi: 10.1186/s40510-014-0031-3.

Marzieh K. et al, Periodontal status of adult patients treated with fixed buccal appliance and removable aligners over one year of active orthodontic treatment, Angle Orthodontist, Volume 83, Issue 1, January 2013

Melsen B., Adult orthodontics, Wiley Blackwell, 2014

Daljit S., Farhad B., Orthodontics. Principles and Practice, Wiley Blackwell, 2011

Szuhanek C. et al,The applications of thermoplastic materials in the fabrication of orthodontic aligners, Materiale plastice, 09/2015; 52(3):385-387Szuhanek C. Mechanical Properties of Welded Orthodontic Metal Appliances, Chapter 24 in DAAAM International Scientific Book 2010, pp. 237-244, B. Katalinic (Ed.), Published by DAAAM International, ISBN 978-3-901509-74-2, ISSN 1726-9687, Vienna, Austria DOI: 10.2507/daaam.scibook.2010.24

Szuhanek, C., Cernescu A., Faur N., Fleser T., Vatau S., Cheveresan A. Numerical simulation of orthodontic forces effects in bone loss cases. Annals of DAAAM for 2010 \& Proceedings of 21th DAAAM International Symposium. Zadar, Croatia, octombrie 2010 